\title{
Improved Approximations for Guarding 1.5-Dimensional Terrains
}

\author{
Khaled Elbassioni • Erik Krohn • \\ Domagoj Matijević • Julián Mestre · \\ Domagoj Ševerdija
}

Received: 16 February 2009 / Accepted: 14 August 2009 / Published online: 2 September 2009

(C) The Author(s) 2009. This article is published with open access at Springerlink.com

\begin{abstract}
We present a 4-approximation algorithm for the problem of placing the fewest guards on a 1.5D terrain so that every point of the terrain is seen by at least one guard. This improves on the previous best approximation factor of 5 (see King in Proceedings of the 13th Latin American Symposium on Theoretical Informatics, pp. 629-640, 2006). Unlike most of the previous techniques, our method is based on rounding the linear programming relaxation of the corresponding covering problem. Besides the simplicity of the analysis, which mainly relies on decomposing the constraint matrix of the LP into totally balanced matrices, our algorithm, unlike previous work, generalizes to the weighted and partial versions of the basic problem.
\end{abstract}

Keywords Terrain guarding $\cdot$ Approximation algorithms $\cdot$ Totally balanced matrices $\cdot$ Geometric covering problems

\section{Introduction}

In the $1.5 D$ terrain guarding problem we are given a polygonal region in the plane determined by an $x$-monotone polygonal chain, and the objective is to find the min-

This paper combines the results of [7] and [19], which were obtained independently: the first gave a 4-approximation for the weighted version of the guarding problem and an extension to partial covering, and the second gave a 4-approximation for the unweighted version. A preliminary version appeared in the Proceedings of the 26th International Symposium on Theoretical Aspects of Computer Science (STACS) 2009.

K. Elbassioni - J. Mestre ( $₫)$

Max-Planck-Institut für Informatik, Saarbrücken, Germany

e-mail: jmestre@mpi-inf.mpg.de

E. Krohn

Department of Computer Science, University of Iowa, Iowa City, USA

D. Matijević · D. Ševerdija

Department of Mathematics, J.J. Strossmayer University of Osijek, Osijek, Croatia 
imum number of guards to place on the chain such that every point in the polygonal region is guarded. A more complete definition of the problem is given is Sect. 2. This kind of guarding problem and its generalizations to 3-dimensions are motivated by optimal placement of antennas for communication networks; for more details see $[1,4]$ and the references therein.

One can easily see that one point is enough to guard the polygonal region if we are allowed to select guards anywhere in the plane. However, the problem becomes interesting if guards can only be placed on the boundary chain. Under this restriction, two natural versions of the problem arise: in the continuous version the guards can be placed anywhere along the chain and all points in the terrain must be guarded, while in the discrete version the guards and points to be guarded are arbitrary subsets of the chain.

\subsection{Previous Work}

Chen et al. [4] claimed that the 1.5D-terrain guarding problem is NP-hard, but a complete proof of the claim was never published [1, 6, 13]. They also gave a linear time algorithm for the left-guarding problem, that is, the problem of placing the minimum number of guards on the chain such that each point of the chain is guarded from its left. Based on purely geometric arguments, Ben-Moshe et al. [1] gave the first constant-factor approximation algorithm for the 1.5D-terrain guarding problem. Although they did not state the value of the approximation ratio explicitly, it was claimed to be at least 6 in [13]. Clarkson et al. [5] gave constant factor approximation algorithms for a more general class of problems using $\epsilon$-nets and showed that their technique can be used to get a constant approximation for the 1.5D-terrain guarding problem. Most recently, King [13] claimed that the problem can be approximated with a factor of 4 , but the analysis turned out to have an error that increases the approximation factor to 5 [14].

\subsection{Our Results and Outline of the Paper}

The main building block of our algorithms is an LP-rounding algorithm for one-sided guarding: a version of the problem where a guard can see either to the left or to the right. Guided by an optimal fractional solution, we can partition the points into those that should be guarded from the left, and those that should be guarded from right. This turns out to be very useful information since we can show that the LPs for the left-guarding and right-guarding problems are integral. We prove this by establishing a connection between the guarding problem and totally balanced covering problems that is of independent interest. Altogether, this leads to a factor 2 approximation for one-sided guarding. Then we show how to reduce other variants of the problem to the one-sided case by incurring an extra multiplicative factor of 2 in the approximation ratio.

A nice feature of this framework is that the algorithms emanating from it, are very simple applications of linear programming and are very simple to analyze. This comes in contrast with the relatively complicated algorithms of $[1,13]$ whose description/analysis involves a fairly long list of cases. In addition, our framework allows us 
to tackle more general versions of the problem than those considered in the literature thus far; for example, guards can have weights and we want to minimize the weight of the chosen guards, or where we are not required to cover all the terrain, but only a prescribed fraction of it. It seems that such variants are very difficult to deal with, if one tries to use only geometric techniques as the ones used in $[1,13]$ for the basic problem. We remark also that, for many geometric set covering problems for which constant factor approximations exist (e.g., covering points in the plane by arbitrary radii disks [3]), it is not clear how to extend these results to the weighted case. So this paper gives one example where such an extension is possible.

It is worth noting that the idea of using the fractional solution to the LP-covering problem to partition the problem into several integral subproblems has been used before $[9,11,20]$.

In the next section, we define the basic guarding problem and its variants more formally. In Sect. 3 we focus on the left guarding problem and show that this is a totally balanced covering problem. Section 4 shows how to get a 2-approximation for one-sided guarding. Finally, in Sect. 5 we apply these results to obtain constant-factor approximation algorithms for more general variants of the guarding problem.

\section{Preliminaries}

A terrain $T$ is an $x$-monotone polygonal chain with $n$ vertices, i.e., a piecewise linear curve intersecting any vertical line in at most one point. Denote by $V$ the vertices of $T$ and by $n=|V|$ the complexity of the chain. The terrain polygon $P_{T}$ determined by $T$ is the closed region in the plane bounded from below by $T$.

For two points $p$ and $q$ in $P_{T}$, we say that $p$ sees $q$ and write $p \sim q$, if the line segment connecting $p$ and $q$ is contained in $P_{T}$, or equivalently, if it never goes strictly below $T$. We will also write $p<q$ if $p$ lies to the left of $q$.

The $1.5 D$-terrain guarding problem for $T$ is to place guards on $T$ such that every point $p \in P_{T}$ is seen by some guard. One can easily see, by the monotonicity of $T$, that any set of guards that guards $T$ is also enough to guard $P_{T}$. Henceforth we restrict our attention to the case when the requirement is to guard all points of $T$.

The continuous $1.5 \mathrm{D}$-terrain guarding problem is to select a smallest set of guards $A \subseteq T$ that sees every point in $T$; in other words, for every $p \in T$ there exists $g \in A$ such that $g \sim p$. We also consider the following variants of this basic problem:

1. In the discrete version we are given a finite set of possible guards $G \subseteq T$ with weights $w: G \rightarrow R^{+}$and a finite set of points $N \subseteq T$. The goal is to select a minimum weight set of guards $A \subseteq G$ to guard $N$.

2. In the partial version we are given a penalty function $p: N \rightarrow \mathbb{R}^{+}$and a budget $b$. The goal is to find a minimum weight set of guards such that the penalty of unguarded points is at most $b$. In the continuous variant, $b$ is the length of $T$ that can be left unguarded.

3. In the one-sided guarding version the guards can see in only one of two directions: left or right. Specifically, given 3 sets of points $N, G_{L}$ and $G_{R}$, we want to find sets $A_{L} \subseteq G_{L}$ and $A_{R} \subseteq G_{R}$ of guards such that for all $p \in N$ there is $g \in A_{L}$ such that $g<p$ and $g \sim p$, or $g \in A_{R}$ such that $g>p$ and $g \sim p$. The sets 
Fig. $1 a<b<c<d$ points and terrain-free region

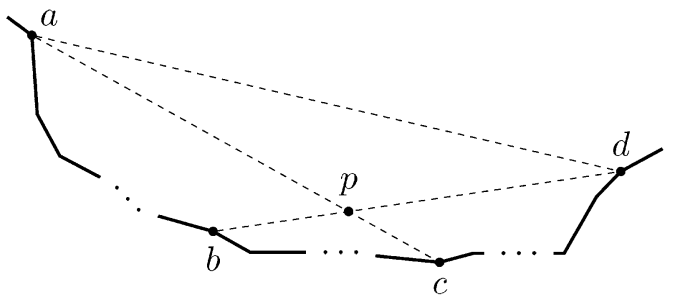

$G_{L}$ and $G_{R}$, and hence $A_{L}$ and $A_{R}$ need not be disjoint. The overall cost of the solution is $w\left(A_{L}\right)+w\left(A_{R}\right)$.

This includes both the left- and right-guarding versions where guards in the given set $G$ can see only from the left, respectively, right (setting $G_{L}=G$ and $G_{R}=\emptyset$ we get the left-guarding problem, while setting $G_{R}=G$ and $G_{L}=\varnothing$ gives the right-guarding problem).

Using a unified framework we get 4-approximations for nearly all ${ }^{1}$ of these variants. Our approach is based on linear programming, totally balanced matrices, and the paradigm of rounding to an integral problem $[9,11]$. We progressively build our approximations by reducing each variant to a simpler problem. First, we start establishing a connection between the left-guarding problem and totally balanced matrices. Then, we show how to use this to get a 2-approximation for the one-sided guarding. Finally, we show how the latter implies a 4-approximation for other variants.

Throughout the paper we will make frequent use of the following property, which was first observed by Ben-Moshe et al. [1]. For the sake of completeness we include a proof here.

Lemma 1 [1] Let $a<b<c<d$ be four points on $T$. If $a \sim c$ and $b \sim d$, then $a \sim d$.

Proof Let $a, b, c, d \in T$ be points such that $a<b<c<d$. Notice that point $b$ must lie below or on $\overline{a c}$ (otherwise, $b$ would break sight between $a$ and $c$ ). Similarly, point $c$ must lie below or on $\overline{b d}$.

Thus, line segments $\overline{a c}$ and $\overline{b d}$ must intersect in at least one point, say $p$ (see Fig. 1). Notice that no point of the terrain can be above $a p$ and $p d$. Therefore, $a \sim d$.

Discussion Lemma 1 will turn out to be of high importance in most of our proofs. Namely, all of the results for the discrete cases in this paper in fact apply to any given set of points and some interaction relation $\sim$ that complies with Lemma 1.

However, it is easy to see that not all points that comply with Lemma 1 can be an instance of the 1.5D-terrain guarding (see Fig. 2).

Let $S(p)=\{g \in G \mid g \sim p\}$ be the set of guards that see point $p \in N$. Denote by $S_{L}(p)=\{g \in G \mid g<p$ and $g \sim p\}$ the set of guards that see $p$ strictly from the left, and analogously by $S_{R}(p)$ the set of guards that see $p$ strictly from the right.

\footnotetext{
${ }^{1}$ The only exception is instances of the discrete variant when $G \cap N \neq \emptyset$. Here we get a 5-approximation.
} 

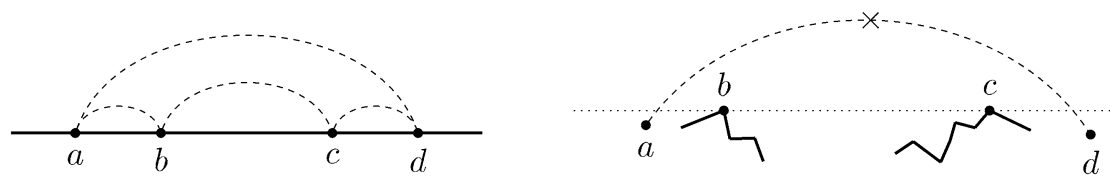

Fig. 2 Let $a, b, c, d$ be points such that $a<b<c<d$ and $a \sim b, b \sim c, c \sim d, a \sim d$ and $a \nsim c, b \nsim d$. Since, $b \sim c$ and $a \neq c$ point $a$ must lie below $\overline{b c}$. By symmetry, point $d$ has to be also below $\overline{b c}$ which gives a geometric contradiction to the fact that $a \sim d$

\section{Left-Guarding and Totally Balanced Matrices}

Even though this section deals exclusively with the left-guarding version, it should be noted that everything said applies, by symmetry, to the right-guarding version. Recall in this case that we are given two sets of points $N, G$, where each point in $N$ has to be guarded using only guards from $G$ that lie strictly to its left.

Consider the following integer linear programming formulation.

$$
\operatorname{minimize} \sum_{g \in G} w_{g} x_{g}
$$

subject to

$$
\begin{aligned}
& \sum_{g \in S_{L}(p)} x_{g} \geq 1 \quad \forall p \in N \\
& x_{g} \in\{0,1\} \quad \forall g \in G
\end{aligned}
$$

Variable $x_{g}$ indicates whether $g$ is chosen as a guard. Constraint (1) asks that every point is seen by some guard from the left. In the following we will show that the solution of the relaxation of (LP1) will always be integral.

Let $A \in\{0,1\}^{|N| \times|G|}$ be a binary matrix. Call $A$ a left-visibility matrix if it corresponds to the guard-point incidence matrix for some instance of the left-guarding problem. Also, $A$ is said to be totally balanced [2] if it does not contain a square submatrix with all row and column sums equal to 2 and no identical columns. Finally, $A$ is in standard greedy form if it does not contain as an induced submatrix

$$
\left[\begin{array}{ll}
1 & 1 \\
1 & 0
\end{array}\right]
$$

An equivalent characterization [12] is that $A$ is totally balanced if and only if $A$ can be put into greedy standard form by permuting its rows and columns.

\section{Lemma 2 Any left-visibility matrix is totally balanced.}

Proof Let $A$ be a left-visibility matrix. We show how to put $A$ into standard greedy form. Permute the rows and columns of A such that the rows from top to bottom correspond to the points ordered from left to right, and the columns from left to right correspond to the guards ordered from right to left. Suppose that there exists an 
induced $2 \times 2$ sub-matrix of the form (2), whose rows are indexed by $p_{1}, p_{2} \in N$, and whose columns are indexed by $g_{1}, g_{2} \in G$. Then we have the following order: $g_{2}<g_{1}<p_{1}<p_{2}$. Now we apply Lemma 1 with $a=g_{2}, b=g_{1}, c=p_{1}$ and $d=p_{2}$ to arrive at the contradiction $p_{2} \sim g_{2}$.

It is known that for a totally balanced matrix $A$, the polyhedron $\{x \geq 0: A x \geq 1\}$ is integral. Furthermore, there is an efficient purely combinatorial algorithm for finding an optimal integral solution to (LP1) due to Kolen [16]. Indeed, in the next subsection we show that this algorithm translates into an extremely simple procedure for the uniform weight case, i.e., when $w_{g}=1$ for all $g \in G$.

\subsection{Uniform Left-Guarding}

For each point $p \in N$ let $L(p)$ denote the left-most guard that sees $p$. Consider the simple greedy algorithm on the set of points $N$ shown below: points in $N$ are scanned from left to right and when we find an unguarded point $p$, we select $L(p)$ as a guard.

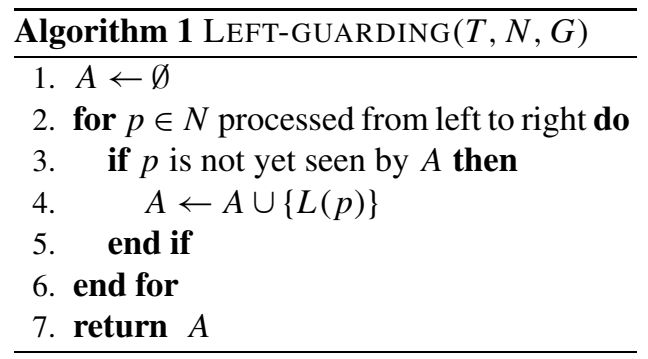

The algorithm can be implemented in $O(|N| \log |G|)$ time using a procedure similar to Graham's scan [10] for convex-hull computation. To see that it returns an optimal solution, let $X \subseteq N$ be those points that force the algorithm to add a guard. Suppose, for the sake of contradiction, that there exist two points $p^{\prime}$ and $p^{\prime \prime}$ in $X$ that are seen from the left by the same guard $g \in G$, in other words, $g<p^{\prime}<p^{\prime \prime}$ and $g \sim p^{\prime}$ and $g \sim p^{\prime \prime}$. Let $g^{\prime}=L\left(p^{\prime}\right)$, and note that $g^{\prime} \leq g$. If $g^{\prime}=g$ then $g^{\prime} \sim p^{\prime \prime}$ and therefore

$p^{\prime \prime}$ would have not been unguarded when it was processed. Hence $g^{\prime}<g$, but Lemma 1 tells us that $g^{\prime} \sim p^{\prime \prime}$ and we get a contradiction. Therefore, each guard in $G$ can see at most one point in $X$, which means $|X|$ is a lower bound on the optimal solution. Since the cardinality of $A$ equals that of $X$, it follows that $A$ is optimum, and hence by, Lemma 2, it returns an optimal solution of (LP1).

Remark Note that in our definition of left-guarding or right-guarding the guard does not see the point on which it lies. The example in Fig. 3 demonstrates that without that condition the polyhedron is not necessarily integral any more.

\section{A 2-Approximation for One-Sided Guarding}

In this section we study discrete weighted one-sided guarding. Recall that in this variant, we are given a set of points $N$ and two sets of guards $G_{L}$ and $G_{R}$, where 


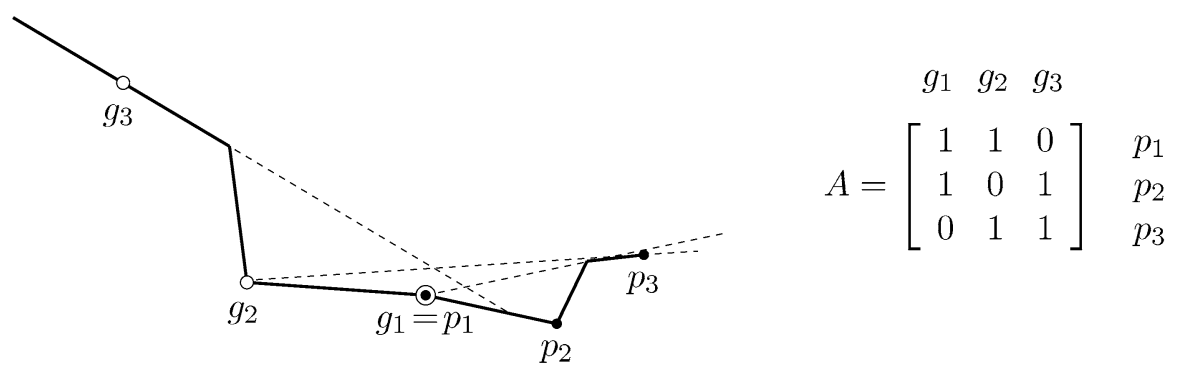

Fig. 3 Consider the set $G=\left\{g_{1}, g_{2}, g_{3}\right\}$ of guards and the set $N=\left\{p_{1}, p_{2}, p_{3}\right\}$ of points as above. Visibility matrix $A$ is shown to the right of the example. Note that the guard $g_{1}$ guards the point on which it lies, i.e. $g_{1}$ guards $p_{1}$. Vertices of the polyhedron $\{x \geq 0: A x \geq 1\}$ are $(0,1,1),(1,1,0),(1,0,1)$ and $(1 / 2,1 / 2,1 / 2)$

each guard in $G_{L}$ (respectively, $G_{R}$ ) can only guard points from $N$ strictly to its right (respectively, strictly to its left). It is easy to construct examples where solving separately the left-guarding and right-guarding problems and then taking the minimum of these two solutions is arbitrarily far from the optimal value. The intuition behind the algorithm is to use the LP solution to determine which points should be guarded from the left and which should be guarded from the right. We assume without loss of generality that each point in $N$ can be seen by a guard on its left or by a guard on its right. Otherwise it must be guarded by itself and the system is infeasible, a situation which can be discovered in a preprocessing step.

We state our main result and then describe the algorithm.

Theorem 1 There is a 2-approximation algorithm for discrete one-sided guarding.

Consider the following LP for finding the optimal set of left and right guards:

$$
\begin{aligned}
& \text { minimize } \sum_{g \in G_{L}} w_{g} x_{g, L}+\sum_{g \in G_{R}} w_{g} x_{g, R} \\
& \text { subject to } \\
& \sum_{g \in S_{L}(p) \cap G_{L}} x_{g, L}+\sum_{g \in S_{R}(p) \cap G_{R}} x_{g, R} \geq 1 \quad \forall p \in N \\
& x_{g, L} \geq 0 \quad \forall g \in G_{L} \\
& x_{g, R} \geq 0 \quad \forall g \in G_{R}
\end{aligned}
$$

Variable $x_{g, L}$ indicates whether $g$ is chosen in $A_{L}$ and $x_{g, R}$ indicates whether $g$ is chosen in $A_{R}$. Constraint (3) asks that every point is seen by some guard, either from the left or from the right.

The algorithm first finds an optimal fractional solution $x^{*}$ to (LP2). Guided by $x^{*}$, we divide the points into two sets

$$
N_{L}=\left\{p \in N \mid \sum_{g \in S_{L}(p) \cap G_{L}} x_{g, L}^{*} \geq \frac{1}{2}\right\}, \quad \text { and }
$$




$$
N_{R}=\left\{p \in N \mid \sum_{g \in S_{R}(p) \cap G_{R}} x_{g, R}^{*} \geq \frac{1}{2}\right\} .
$$

Using the results from Sect. 3, we solve optimally the left-guarding problem for the pair $\left(N_{L}, G_{L}\right)$ and the right-guarding problem for the pair $\left(N_{R}, G_{R}\right)$. This gives us two sets of guards $A_{L}^{*}$ and $A_{R}^{*}$. The final solution is a combination of these two.

The fractional solution allows us to bound the cost of $A_{L}^{*}$ and $A_{R}^{*}$.

Lemma 3 Let $A_{L}^{*}$ and $A_{R}^{*}$ be optimal solutions for the pairs $\left(N_{L}, G_{L}\right)$ and $\left(N_{R}, G_{R}\right)$ respectively. Then $w\left(A_{L}^{*}\right) \leq 2 \sum_{g \in G_{L}} w_{g} x_{g}^{*}$ and $w\left(A_{R}^{*}\right) \leq 2 \sum_{g \in G_{R}} w_{g} x_{g}^{*}$.

Proof We only prove the first inequality as the second is symmetrical. Setting $x_{g, L}=2 x_{g}^{*}$ we get a fractional solution for (LP1) for guarding $N_{L}$. The solution $x$ is feasible, by definition of $N_{L}$, and its cost is $2 \sum_{g \in G_{L}} w_{g} x_{g}^{*}$. Therefore, the optimal fractional solution can only be smaller than that. Lemma 2 tells us that the cost of an optimal fractional solution is the same as the cost of an optimal integral solution, namely, $w\left(A_{L}^{*}\right)$.

Since $\sum_{g \in G_{L}} w_{g} x_{g, L}^{*}+\sum_{g \in G_{R}} w_{g} x_{g, R}^{*}$ is a lower bound on the cost of an optimal solution for guarding $N$, it follows that the cost of $\left(A_{L}^{*}, A_{R}^{*}\right)$ is at most twice the optimum. To see that this is feasible, consider some point $p \in N$. Because of (3) and our assumption that each point is seen by some guard on its left or on its right, it must be the case that $p \in N_{L}$ or $p \in N_{R}$. Therefore $p$ must be covered, either from the left by $A_{L}^{*}$ or from the right by $A_{R}^{*}$.

To compute $A_{L}^{*}$ and $A_{R}^{*}$ we can take the fractional solution to (LP1) and turn it into a basic, and therefore integral, solution without increasing its cost. Alternatively, we can run Kolen's algorithm [16] for matrices in greedy standard form. This finishes the proof of Theorem 1 .

\subsection{Partial Covering}

In this section we focus on the partial version of the one-sided guarding problem.

Theorem 2 There is a polynomial $(2+\epsilon)$-approximation and a quasi-polynomial time 2-approximation for partial discrete one-sided guarding.

Our approach is based on the framework of Mestre [20]. We say $A$ is a one-sided-visibility matrix if it is the guard-point incidence matrix of the covering problem defined by (LP2) for some instance of the one-sided guarding problem. Also, $A$ is said to be 2-separable if there exist binary matrices $A_{1}$ and $A_{2}$ such that $A=A_{1}+A_{2}$ and every matrix $B$ formed by taking rows from $A_{1}$ or $A_{2}$ is totally

balanced (the $i$ th row of $B$ is the $i$ th row of $A_{1}$ or the $i$ th row of $A_{2}$, for all $i$ ).

Proposition 1 [20] Let $A$ be a 2-separable matrix. Then there is a $(2+\epsilon)$ approximation and a quasi-polynomial time 2-approximation for the partial problem defined by $A$. 
Therefore, all we need to do to prove Theorem 2 is to argue that every one-sided visibility matrix is 2-separable.

Lemma 4 Any one-sided visibility matrix is 2-separable.

Proof Let $A$ be a one-sided visibility matrix and assume, without loss of generality, that $A$ has the form $\left[C_{1} C_{2}\right]$ where the columns of $C_{1}$ correspond to left guards $G_{L}$ and the columns of $C_{2}$ correspond to the right guards $G_{R}$.

Our decomposition of $A$ uses $A_{1}=\left[C_{1} 0\right]$ and $A_{2}=\left[0 C_{2}\right]$. Suppose that a matrix $B$ is formed by taking rows from $A_{1}$ and $A_{2}$. Let $N_{L}$ be the set of rows originating

from $A_{1}$ and $N_{R}$ the set of rows originating from $A_{2}$ (note that $N_{L}$ and $N_{R}$ constitute a partition of $N$ ). Permute the rows of $B$ so that rows in $N_{L}$ appear before rows in $N_{R}$. This gives rise to the following block matrix

$$
B^{\prime}=\left[\begin{array}{cc}
D_{1} & 0 \\
0 & D_{2}
\end{array}\right]
$$

where the rows of $D_{1}$ correspond to points in $N_{L}$ and its columns to left guards, and the rows of $D_{2}$ correspond to points in $N_{R}$ and its columns to right guards. By Lemma 2 both $D_{1}$ and $D_{2}$ are totally balanced. Therefore we can permute the rows and columns of $B^{\prime}$ to get a new matrix

$$
B^{\prime \prime}=\left[\begin{array}{cc}
D_{1}^{\prime} & 0 \\
0 & D_{2}^{\prime}
\end{array}\right]
$$

where $D_{1}^{\prime}$ and $D_{2}^{\prime}$ are in standard greedy form, which in turn implies that $B^{\prime \prime}$ is also in standard greedy form. It follows that $B^{\prime \prime}, B^{\prime}$, and $B$ are totally balanced.

This finishes the proof of Theorem 2 .

\section{Applications}

In this section we show how to use the 2-approximations for one-sided guarding to design good approximation algorithms for more general variants.

\subsection{The Continuous Case}

We assume for simplicity that the weights are uniform. Recall that in this variant guards can be placed anywhere on the terrain and we are to guard all the points. We will reduce the problem to the discrete case, where $G \cap N=\emptyset$. Our reduction follows the approach of Ben-Moshe et al. [1].

Theorem 3 There is a 4-approximation algorithm for the continuous case and a $(4+\epsilon)$-approximation for its partial version. 
Fig. 4 Example of additional set of points/guards for a vertex $v$ of $T$. Point $p$ is the point selected from the essential segment $\overline{a b}$

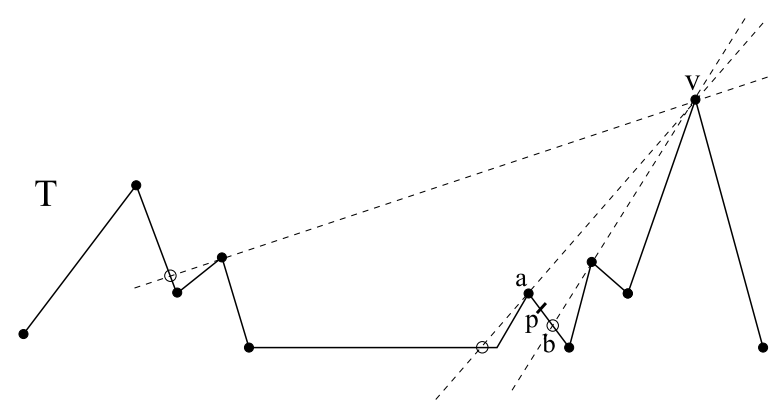

Let $A^{*}$ be an optimum set of guards for a given instance $T$ of the continuous problem. Consider a guard $g$ in $A^{*}$. If $g$ is not a vertex of $T$ then it must lie on a segment $\overline{p q}$ of $T$. Suppose without loss of generality that $p<q$, then a left guard at $p$ and a right guard at $q$ can see at least as much as $g$ does. If $g$ is a vertex of $T$ then a left guard and a right guard at $g$ together can see the same as $g$ does minus $g$ itself.

Therefore there exists a solution $A^{\prime}$ that uses only left and right guards on the vertices of $T$ that covers $T \backslash V$ such that $\left|A^{\prime}\right|=2\left|A^{*}\right|$.

To deal with the fact that every point must be guarded, consider the line through each pair of vertices $v_{1}, v_{2} \in V$ such that $v_{1} \sim v_{2}$ and introduce at most two new points that see $v_{1}$ and $v_{2}$ at the place where the line intersects the terrain. These points partition $T$ into $O\left(n^{2}\right)$ essential segments. In the strict interior of each segment introduce an additional point $p$ that is responsible for the segment. Let $M$ be the set of all such points. (See Fig. 4 for an illustration.) The key realization is that for every guard $g \in V$ and essential segment $\overline{a b}$, either $g$ can see the whole segment or nothing of it.

Hence, a feasible solution to the one-sided discrete version with $G_{L}=G_{R}=V$ and $N=M$ also constitutes a feasible solution to the continuous case. Let $A^{\prime \prime}$ be an optimal solution for this discrete problem, and $A^{\prime \prime \prime}$ be the solution returned by Theorem 1. Since $A^{\prime}$ is feasible for the discrete instance, we get $\left|A^{\prime \prime \prime}\right| \leq 2\left|A^{\prime \prime}\right| \leq 2\left|A^{\prime}\right|=4\left|A^{*}\right|$ and we get an overall approximation factor of 4 . For the partial version where we want at most a fraction of the length to be left unguarded we give to each point in $p \in M$ a penalty equal to the length of the essential segment it is responsible for.

In a more realistic setting, different parts of the terrain could have different penalties. A possible complication in this case is how to specify the penalty function (after all, the terrain is continuous). We note that as long as we have an oracle to compute the penalty of the essential segments, the same approach from above should work for non-uniform penalty functions in the continuous case.

Remark The assumption that the weights are uniform can be relaxed using standard discretization techniques at the expense of a small increase in the approximation factor. Indeed, consider a segment $\overline{p q}$ and assume that the weight function $w(x)$ for $x \in \overline{p q}$, has $r$ local minima in this interval. Then each segment $\overline{s t} \subseteq \overline{p q}$ on which the function either monotonically increases (or monotonically decreases), say from $t_{1}$ to $t_{2}$ (respectively, from $t_{2}$ to $t_{1}$ ), can be divided into $\log _{1+\epsilon}\left(t_{2} / t_{1}\right)$ sub-segments, 
where $\epsilon>0$ is an arbitrarily small constant. Now, if there is an optimal guard on any of these sub-segments, it can be replaced by a left-guard and a right-guard at the end-points of the sub-segment whose combined weight is at most $(2+\epsilon)$ times the weight of the original guard (in the uniform case we only pay 2). This results in an increase in the number of candidate guards by a factor of at most $2 r \log _{1+\epsilon}\left(t_{2} / t_{1}\right)$ per $\overline{p q}$ segment, and a loss of an additional $1+\epsilon$ in the overall approximation factor.

\subsection{The Discrete Case}

We consider the discrete version where we are given a set of guards $G$ and set of points $N$ to guard. In this case, guards can see in both directions.

Theorem 4 There is a 4-approximation for the weighted discrete case and $(4+\epsilon)$ approximation for its partial version when $G \cap N=\emptyset$. Otherwise, we get 5 and $(5+\epsilon)$-approximations respectively.

The case where $G \cap N=\emptyset$ is easily handled by replacing a guard that can see in both directions with a left guard and a right guard. Thus we pay a factor 2 to reduce the general problem to one-sided guarding. This also holds for the partial version.

Notice that if $G \cap N \neq \emptyset$ then the reduction above must pay a factor of 3 since a point guarding itself must be guarded by some other point strictly from the left or the right, and thus it only leads to a 6-approximation. To get the ratio of 5 we need to use yet another linear program.

$$
\operatorname{minimize} \sum_{g \in G} w_{g} x_{g}
$$

subject to

$$
\begin{array}{ll}
\sum_{g \in S(p)} x_{g} \geq 1 & \forall p \in N \\
x_{g} \geq 0 & \forall g \in G
\end{array}
$$

Let $x^{*}$ be an optimal fractional solution to (LP3). As in the one-sided case we will let the solution $x$ dictate which points should be self-guarded and which should be guarded by others. Define

$$
A_{0}=\left\{g \in N \cap G \mid x_{g}^{*} \geq \frac{1}{5}\right\} .
$$

We place guards at $A_{0}$ at a cost of most

$$
w\left(A_{0}\right) \leq 5 \sum_{g \in A_{0}} w_{g} x_{g}^{*}
$$

Let $N^{\prime}$ be the set of points in $N$ not seen by $A_{0}$ and let $G^{\prime}=G \backslash A_{0}$. We will construct a fractional solution for the one-sided guarding problem for $N^{\prime}$ and 
$G_{L}=G_{R}=G^{\prime}$. For each $g \in G^{\prime}$ let $x_{g, L}=x_{g, R}=\frac{5}{4} x_{g}^{*}$. The fractional solution $x$ is feasible for (LP2) since for all $p \in N^{\prime}$

$$
\sum_{g \in S_{L}(p) \cap G_{L}} x_{g, L}+\sum_{g \in S_{R}(p) \cap G_{R}} x_{g, R}=\frac{5}{4} \sum_{g \in S(p) \backslash\{p\}} x_{g}^{*} \geq \frac{5}{4}\left(1-\frac{1}{5}\right)=1
$$

Let $\left(A_{L}^{*}, A_{R}^{*}\right)$ be the solution found for the one-sided problem. The cost of these sets of guards is guaranteed to be at most twice that of $x$, which in turn is

$\frac{5}{2} \sum_{g \in G \backslash A_{0}} w_{g} x_{g}^{*}$. Thus the overall cost is

$$
w\left(A_{L}^{*}\right)+w\left(A_{R}^{*}\right) \leq 5 \sum_{g \in G \backslash A_{0}} w_{g} x_{g}^{*}
$$

Hence, the second part of Theorem 4 follows from (4) and (5). Finally, for the partial version we note the proof of Proposition 1 uses as a lower bound the cost of the optimal fractional solution, so the cost of the solution returned can still be related to the cost of $x$, which is necessary to get the stated approximation guarantee.

\section{Concluding Remarks}

We gave a 4-approximation for the continuous 1.5D terrain guarding problem as well as several variations of the basic problem. Our results rely, either explicitly or implicitly, on the LP formulation (LP3) for the discrete case. For the unweighted version of the problem, there is a very simple $O(|N| \log |G|)$-algorithm for solving

the left-guarding LP (LP1). Furthermore, at the loss of factor $(1+\epsilon)$ in the approximation ratios, one can use fast techniques for covering LPs (see e.g. $[8,21]$ )

to solve (LP2). In particular, using the recent results of Koufogiannakis and

Young [17, 18], a $(1+\epsilon)$-approximation for (LP2) can be found in time $O\left(|N||G| \log (|N|+|G|) / \epsilon^{2}\right)$, which is only a polylogarithmic factor slower than the purely combinatorial algorithms for the uniform weight case (see e.g. [13]).

Very recently, King [15] showed that the VC dimension of the discrete case is exactly 4 . More precisely, he showed a terrain with 4 guards and 16 points (these sets are disjoint) such that each point is seen by a different subset of the guards. If we have to cover the points that are seen by pairs of guards, we get precisely a vertex cover problem on the complete graph with 4 vertices. An integral solution must pick 3 vertices, while a fractional solution can pick a half of all vertices. It follows that the integrality gap of (LP3) is at least $3 / 2$, even when $G \cap N=\emptyset$. On the other hand, our analysis shows that the gap is at most 4 . We leave as an open problem to determine the exact integrality gap of (LP3).

Acknowledgements We would like to thank Jamie King and Kasturi Varadarajan for their valuable comments. The fourth author was supported by an Alexander von Humboldt Fellowship.

Open Access This article is distributed under the terms of the Creative Commons Attribution Noncommercial License which permits any noncommercial use, distribution, and reproduction in any medium, provided the original author(s) and source are credited. 


\section{References}

1. Ben-Moshe, B., Katz, M.J., Mitchell, J.S.B.: A constant-factor approximation algorithm for optimal 1.5D terrain guarding. SIAM J. Comput. 36(6), 1631-1647 (2007)

2. Berge, C.: Balanced matrices. Math. Program. 2, 19-31 (1972)

3. Brönnimann, H., Goodrich, M.T.: Almost optimal set covers in finite VC-dimension. Discrete Comput. Geom. 14(4), 463-479 (1995)

4. Chen, D.Z., Estivill-Castro, V., Urrutia, J.: Optimal guarding of polygons and monotone chains. In: Proceedings of the 7th Canadian Conference on Computational Geometry, pp. 133-138 (1995)

5. Clarkson, K.L., Varadarajan, K.R.: Improved approximation algorithms for geometric set cover. In: Proceedings of the 20th Symposium on Computational Geometry, pp. 135-141 (2005)

6. Demaine, E.D., O'Rourke, J.: Open problems: open problems from CCCG 2005. In: Proceedings of the 18th Canadian Conference on Computational Geometry, pp. 75-80 (2006)

7. Elbassioni, K., Matijević, D., Mestre, J., Ševerdija, D.: Improved approximations for guarding 1.5-dimensional terrains. CoRR, abs/0809.0159v1 (2008)

8. Garg, N., Könemann, J.: Faster and simpler algorithms for multicommodity flow and other fractional packing problems. In: 39th Annual IEEE Symposium on Foundations of Computer Science (FOCS), pp. 300-309 (1998)

9. Gaur, D.R., Ibaraki, T., Krishnamurti, R.: Constant ratio approximation algorithms for the rectangle stabbing problem and the rectilinear partitioning problem. J. Algorithms 43(1), 138-152 (2002)

10. Graham, R.L.: An efficient algorithm for determining the convex hull of a finite planar set. Inf. Process. Lett. 1(4), 132-133 (1972)

11. Hassin, R., Segev, D.: Rounding to an integral program. Oper. Res. Lett. 36(3), 321-326 (2008)

12. Hoffman, A.J., Kolen, A., Sakarovitch, M.: Totally-balanced and greedy matrices. SIAM J. Algebr. Discrete Methods 6, 721-730 (1985)

13. King, J.: A 4-approximation algorithm for guarding 1.5-dimensional terrains. In: Proceedings of the 13th Latin American Symposium on Theoretical Informatics, pp. 629-640 (2006)

14. King, J.: Errata on "A 4-approximation algorithm for guarding 1.5-dimensional terrains". http://www.cs.mcgill.ca/ jking/papers/4apx_latin.pdf

15. King, J.: VC-dimension of visibility on terrains. In: Proceedings of the 20th Canadian Conference on Computational Geometry, pp. 27-30 (2008)

16. Kolen, A.: Location problems on trees and in the rectilinear plane. Ph.D. thesis, Matematisch Centrum, Amsterdam (1982)

17. Koufogiannakis, C., Young, N.E.: Beating simplex for fractional packing and covering linear programs. In: 48th Annual IEEE Symposium on Foundations of Computer Science (FOCS), pp. 494-504 (2007)

18. Koufogiannakis, C., Young, N.E.: Beating simplex for fractional packing and covering linear programs. CoRR, abs/0801.1987 (2008)

19. Krohn, E.: Survey of terrain guarding and art gallery problems. Unpublished manuscript. (November 2007)

20. Mestre, J.: Lagrangian relaxation and partial cover (extended abstract). In: Proceedings of the 25th Annual Symposium on Theoretical Aspects of Computer Science, pp. 539-550 (2008)

21. Plotkin, S.A., Shmoys, D.B., Tardos, É.: Fast approximation algorithms for fractional packing and covering problems. In: 32nd Annual IEEE Symposium on Foundations of Computer Science (FOCS), pp. 495-504 (1991) 